\title{
INSENSITIVE BOUNDS FOR THE MOMENTS OF THE SOJOURN TIMES IN M/GI SYSTEMS UNDER STATE-DEPENDENT PROCESSOR SHARING
}

\author{
ANDREAS BRANDT, ${ }^{*}$ Humboldt-Universität zu Berlin \\ MANFRED BRANDT, ${ }^{* *}$ Zuse Institute Berlin
}

\begin{abstract}
We consider a system with Poisson arrivals and independent and identically distributed service times, where requests in the system are served according to the state-dependent (Cohen's generalized) processor-sharing discipline, where each request receives a service capacity that depends on the actual number of requests in the system. For this system, we derive expressions as well as tight insensitive upper bounds for the moments of the conditional sojourn time of a request with given required service time. The bounds generalize and extend corresponding results, recently given for the single-server processor-sharing system in Cheung et al. (2006) and for the state-dependent processorsharing system with exponential service times by the authors (2008). Analogous results hold for the waiting times. Numerical examples for the M/M/m-PS and M/D/m-PS systems illustrate the given bounds.
\end{abstract}

Keywords: Poisson arrival; general service time; state-dependent processor sharing; Cohen's generalized processor sharing; conditional sojourn time; conditional waiting time; moments; insensitive bounds; many server; M/GI/m-PS; Laplace-Stieltjes transform

2000 Mathematics Subject Classification: Primary 60K25; 68M20; 90B22;60E15;

$60 \mathrm{G} 10$

\section{Introduction}

Processor-sharing (PS) systems have been widely used in the last decades for modeling and analyzing computer and communication systems; see, e.g. [3], [4], [6], [10], [12], [13], [14], [16], [22], [25], and the references therein. In this paper we deal with insensitive bounds for the moments of sojourn times of the following PS service system, denoted by M/GI/SDPS. At a node requests arrive according to a Poisson process of intensity $\lambda$ with independent and identically distributed service times, which are independent of the arrival process and have the distribution function (DF) $B(x):=\mathrm{P}(S \leq x)$, where $S$ denotes a generic service time with finite positive mean $m_{S}:=\mathrm{E} S$. Note that $B(0)>0$ is allowed, i.e. that zero service times may occur. The requests are served according to the following state-dependent processor-sharing (SDPS) discipline (Cohen's generalized processor-sharing discipline); see [3] and [11]. (The SDPS discipline seems to go back to Cohen (cf. [11]), who referred to it as the generalized processorsharing discipline. But nowadays this term is used for other classes of models, such as weighted fair queueing systems. Therefore, it is now called the SDPS discipline; cf., e.g. [3].) If there are

Received 9 January 2009; revision received 3 November 2009.

* Postal address: Institut für Operations Research, Humboldt-Universität zu Berlin, Spandauer Strasse 1, D-10178 Berlin, Germany. Email address: brandt@wiwi.hu-berlin.de

** Postal address: Konrad-Zuse-Zentrum für Informationstechnik Berlin (ZIB), Takustrasse 7, D-14195 Berlin, Germany. Email address: brandt@zib.de 
$n \in \mathbb{N}:=\{1,2, \ldots\}$ requests in the node then each of them receives a positive service capacity $\varphi(n)$, i.e. during an interval of length $\Delta \tau$ each of the $n$ requests receives a $\varphi(n) \Delta \tau$ amount of service. In the case in which $\varphi_{1}(n)=1 / n, n \in \mathbb{N}$, we obtain the well known M/GI/1-PS system (single-server system with egalitarian processor-sharing discipline); see [10], [21], and [23]. In the case in which $\varphi_{1, k}(n)=1 /(n+k), n \in \mathbb{N}$, we have a single-server PS system with $k \in \mathbb{N}$ permanent requests in the system; see [18]. In the case in which $\varphi_{m}(n)=\min (m / n, 1), n \in \mathbb{N}$, we have an M/GI/m-PS system; i.e. an $m$-server PS system, where all requests are served in a PS mode, but each request receives at most the capacity of one processor; see [11, p. 283], [5], and [12]. In the case in which $\varphi_{m, k}(n)=\min (m /(n+k), 1), n \in \mathbb{N}$, we have an $m$-server PS system with $k \in \mathbb{N}$ permanent requests. In the case in which $\varphi(n)=1, n \in \mathbb{N}$, the system corresponds to an $\mathrm{M} / \mathrm{GI} / \infty$ system.

Networks with nodes working under the SDPS discipline were investigated in [2], [6], [7], [8], [11], and [24]. In particular, for the M/GI/SDPS system, some basic results are known (see [11]), which we will use and shortly review in the following. Let $N(t)$ be the number of requests in the system at time $t$, and let $R^{*}(t):=\left(R_{1}^{*}(t), \ldots, R_{N(t)}^{*}(t)\right)$ be the vector of the residual service times of the $N(t)$ requests in the system at time $t$, ordered randomly. The vector process $\left(N(t) ; R^{*}(t)\right), t \in \mathbb{R}$, is a Markov process. The M/GI/SDPS system is stable, i.e. there exists a unique stationary process $\left(N(t) ; R^{*}(t)\right), t \in \mathbb{R}$, if and only if

$$
\sum_{n=0}^{\infty} \prod_{\ell=1}^{n} \frac{\varrho \chi(\ell)}{\ell}<\infty
$$

where $\chi(n):=1 / \varphi(n), n \in \mathbb{N}$, and $\varrho:=\lambda m_{S}$ denotes the offered load; cf. [11, Equation (7.18)]. We assume in the following that the system is stable and in a steady state, i.e. that (1.1) is fulfilled and that $\left(N(t) ; R^{*}(t)\right), t \in \mathbb{R}$, is a stationary Markov process. Then the stationary occupancy distribution $p(n):=\mathrm{P}(N(t)=n), n \in \mathbb{Z}_{+}$, and $\mathrm{P}\left(N(t)=n ; R_{1}^{*}(t) \leq\right.$ $\left.r_{1}, \ldots, R_{n}^{*}(t) \leq r_{n}\right), n \in \mathbb{Z}_{+}, r_{1}, \ldots, r_{n} \in \mathbb{R}_{+}$, i.e. the stationary distribution of $\left(N(t) ; R^{*}(t)\right)$ on $\{N(t)=n\}$, are given by

$$
\begin{gathered}
p(n)=\left(\sum_{m=0}^{\infty} \prod_{\ell=1}^{m} \frac{\varrho \chi(\ell)}{\ell}\right)^{-1} \prod_{\ell=1}^{n} \frac{\varrho \chi(\ell)}{\ell}, \\
\mathrm{P}\left(N(t)=n ; R_{1}^{*}(t) \leq r_{1}, \ldots, R_{n}^{*}(t) \leq r_{n}\right)=p(n) \prod_{\ell=1}^{n} B_{R}\left(r_{\ell}\right),
\end{gathered}
$$

where

$$
B_{R}(x):=\frac{1}{m_{S}} \int_{0}^{x}(1-B(t)) \mathrm{d} t, \quad x \in \mathbb{R}_{+},
$$

denotes the stationary residual service time distribution having the density $b_{R}(x)=(1-$ $B(x)) / m_{S}, x \in \mathbb{R}_{+}$; see [11, Equation (7.19)] for the case of phase-type distributed service times and [24] for the general case. For the sojourn time $V$ of an arbitrary arriving request with required service time $S$, from Little's law and (1.2), we find that

$$
\mathrm{E} V=\frac{1}{\lambda} \sum_{n=1}^{\infty} n p(n)=m_{S} \sum_{n=0}^{\infty} \chi(n+1) p(n) .
$$

For $\tau \in \mathbb{R}_{+}$, let $V(n, \tau)$ be the sojourn time of a tagged arriving request with required service time $\tau$ (abbreviated to $\tau$-request) finding $n$ requests at its arrival in the system, and let $V(\tau)$ be 
the sojourn time of a tagged arriving $\tau$-request. For $V(\tau)$, it is stated that

$$
\mathrm{E} V(\tau)=\frac{\tau}{m_{S}} \mathrm{E} V
$$

cf. [11, Equation (7.27)]. It seems that in the case of the general M/GI/SDPS system, for $V$ and $V(\tau)$, besides (1.5) and (1.6), asymptotic results are known only for heavy-tailed service times; cf. [12]. However, for special cases, several analytical results and numerical algorithms are known. We mention only a few references. For the M/GI/1-PS system and special cases, see, e.g. [10], [13], [18], [21], [22], and [23]. The M/M/2-PS system is treated in [17] and the $\mathrm{M} / \mathrm{M} / \mathrm{m}-\mathrm{PS}$ system is treated in [5]. For the general M/M/SDPS system, see [6].

The aim of this paper is to derive for the M/GI/SDPS system tight insensitive upper bounds for the moments of $V(\tau)$, which generalize corresponding results, recently given for the M/GI/1PS system by [9] and for the M/M/SDPS system by the authors [6], as well as expressions for the Laplace-Stieltjes transforms (LSTs) and moments of $V(\tau)$ and $V$, respectively, which are useful for deriving explicit and numerically treatable representations in some special cases; see Example 2.1, below, and, in particular, [8]. The tight insensitive upper bounds for the moments of $V(\tau)$ are summarized in the following theorem, which will be proved in Section 3.

Theorem 1.1. Let the stability condition (1.1) for the M/GI/SDPS system be satisfied. Then, for $k \in \mathbb{Z}_{+}$, the kth moment of $V(\tau), \tau \in \mathbb{R}_{+}$, is finite if

$$
\sum_{n=0}^{\infty} \chi(n+1)^{k} p(n)<\infty
$$

For $k \in \mathbb{Z}_{+}$, it holds that

$$
\begin{gathered}
\tau^{k}\left(\sum_{n=0}^{\infty} \chi(n+1) p(n)\right)^{k} \leq \mathrm{E}\left[V^{k}(\tau)\right] \leq \tau^{k} \sum_{n=0}^{\infty} \chi(n+1)^{k} p(n), \quad \tau \in \mathbb{R}_{+}, \\
\lim _{\tau \downarrow 0} \frac{\mathrm{E}\left[V^{k}(\tau)\right]}{\tau^{k}}=\sum_{n=0}^{\infty} \chi(n+1)^{k} p(n) .
\end{gathered}
$$

There exists a family of service time distributions $B_{q}(x), q \in(0,1]$, with the given mean $m_{S}$ such that, for the sojourn times $V_{q}(\tau)$ of a tagged arriving $\tau$-request in the M/GI/SDPS system with service time distribution $B_{q}(x)$, it holds that

$$
\lim _{q \downarrow 0} \mathrm{E}\left[V_{q}^{k}(\tau)\right]=\tau^{k} \sum_{n=0}^{\infty} \chi(n+1)^{k} p(n), \quad \tau \in \mathbb{R}_{+}, k \in \mathbb{Z}_{+} .
$$

If, in addition, (1.7) is satisfied then it holds that

$$
\lim _{\tau \downarrow 0} \frac{\mathrm{E}\left[V^{k}(n, \tau)\right]}{\tau^{k}}=\chi(n+1)^{k}, \quad n \in \mathbb{Z}_{+} .
$$

Remark 1.1. The results of Theorem 1.1 are insensitivity results with respect to the service time distribution for given $m_{S}$. Note that in the case in which $k=1$, from Cohen's result (1.6), we know that (1.8) holds; cf. also the discussion after the proof of Theorem 3.1, below. In the case in which $k>1$ the right-hand side of (1.8) provides an insensitive upper bound for $\mathrm{E}\left[V^{k}(\tau)\right]$, which is best possible due to (1.10), and, for small positive values of $\tau$, a good approximation for $\mathrm{E}\left[V^{k}(\tau)\right]$ because of (1.9). 
For the M/M/SDPS system and waiting times, Theorem 1.1 was recently proved in [6]; see Theorem 2.1 therein. For the M/GI/1-PS system, i.e. for $\varphi_{1}(n)=1 / n, n \in \mathbb{N}$, Theorem 1.1 was proved in [9] (see their Theorems 4.1 and 5.11) using stochastic ordering theory and particular results known for M/GI/1-PS systems. Analogously to [9] for the M/GI/1-PS system, the right-hand side of (1.8) can be interpreted as follows. Consider a tagged $\tau$-request finding at its arrival $n$ requests in the system. If during the service of the $\tau$-request no arrival and no departure occurs, then $\hat{V}(\tau)=\tau \chi(n+1)$ is the sojourn time of the $\tau$-request, and its $k$ th moment is $\hat{V}^{k}(\tau)=\tau^{k} \chi(n+1)^{k}$. Thus, if $\tau$ is small then $\hat{V}(\tau)=\tau \chi(N(t)+1)$ is approximately the sojourn time of a $\tau$-request, and, therefore, $\hat{V}(\tau)$ is called in [9] the instantaneous sojourn time as $\tau \downarrow 0$. Note that the right-hand side of (1.8) is just the $k$ th moment of the instantaneous sojourn time $\hat{V}(\tau)$, i.e. $\mathrm{E}\left[\hat{V}^{k}(\tau)\right]$ is for all $\tau \in \mathbb{R}_{+}$an upper bound for $\mathrm{E}\left[V^{k}(\tau)\right]$, and, thus, Theorem 1.1 generalizes the result of [9] to the general M/GI/SDPS system. In the case of a stable M/GI/1-PS system, assumption (1.7) is satisfied for all $k \in \mathbb{N}$, in view of $p(n)=(1-\varrho) \varrho^{n}, n \in \mathbb{Z}_{+}$, and $\varrho<1$, and, hence, all moments of $V(\tau)$ are finite. However, for a stable M/GI/SDPS system, (1.7) is not fulfilled in general, e.g. in the case of $\varphi(n):=(n+k+1) / n^{2}, n \in \mathbb{N}$, and $\varrho:=1$ for $k \in \mathbb{N}$.

The paper is organized as follows. In Section 2 we first derive a linear system of partial differential equations (PDEs) for the LSTs of the sojourn time of a request under the condition that the residual service times of the other requests in the system are also given. Then we derive preliminary results (Lemma 2.1), needed later. Although an explicit solution of the PDEs can be given only for special cases, the LSTs for the sojourn time $V(n, \tau)$ of a $\tau$-request, finding $n$ requests at its arrival in the system, as well as for $V(\tau)$ and for $V$, can be given in terms of the solution of the PDEs (Theorem 2.1 and Corollary 2.1). In Section 3 we first prove Theorem 1.1 using Hölder's inequality and the results of Section 2. Furthermore, relations are given for the moments of sojourn times (Theorem 3.1, Theorem 3.2, and Remark 3.1), which reduce the numerical complexity of computing sojourn time characteristics in some special cases; see [6] and [8]. All results proved in the paper have a correspondence to results for waiting times. In Section 4 we summarize a few of them. Finally, in Section 5 we provide numerical examples for $\mathrm{M} / \mathrm{M} / \mathrm{m}-\mathrm{PS}$ and $\mathrm{M} / \mathrm{D} / \mathrm{m}-\mathrm{PS}$ systems illustrating the bounds for the second moment of $V(\tau)$ given in Theorem 1.1.

\section{Sojourn times and preliminary results}

As mentioned above, we assume in the following that the system is stable, i.e. that (1.1) is fulfilled, and in the steady state. In particular, $m_{S}$ is finite. Moreover, for technical reasons, unless stated otherwise, we make the following assumption:

(A1) $B(x)$ has a continuous density and $B(x)<1$ for $x \in \mathbb{R}_{+}$.

For notational convenience, let $b(x):=\mathrm{d} B(x) / \mathrm{d} x$ be the density of $B(x)$, and let $\bar{B}(x):=$ $1-B(x), \bar{B}_{R}(x):=1-B_{R}(x)$ (cf. (1.4)) and $\beta(x):=b(x) / \bar{B}(x), \beta_{R}(x):=b_{R}(x) / \bar{B}_{R}(x)$ be the complementary distributions and hazard rates of the service time DF and the stationary residual service time DF, respectively. Furthermore, we will use some vector notation. Unless stated otherwise, let $r:=\left(r_{1}, \ldots, r_{\ell}\right) \in \mathbb{R}_{+}^{\ell}$, where $\ell=m$ or $\ell=n$, respectively, and

$$
\Omega_{\ell}:=\left\{r \in \mathbb{R}_{+}^{\ell}: 0<r_{1}<\cdots<r_{\ell}\right\} .
$$

For $x, y \in \mathbb{R}^{\ell}$, let $x \leq y$ if and only if $x_{i} \leq y_{i}$ for $i=1, \ldots, \ell$.

Besides the randomly ordered residual service times $R_{1}^{*}(t), \ldots, R_{N(t)}^{*}(t)$, we need to order the residual service times increasingly. Let $0 \leq R_{1}(t) \leq \cdots \leq R_{N(t)}(t)$ be the residual service 
times of the $N(t)$ requests at time $t$, ordered increasingly, and let $R(t):=\left(R_{1}(t), \ldots, R_{N(t)}(t)\right)$ be the corresponding vector. In view of the SDPS discipline, this implies that the requests are also ordered according to their departure times. For $n \in \mathbb{N}$ and $r \in \Omega_{n}$, let

$$
p(n ; r):=\frac{\partial^{n}}{\partial r_{1} \cdots \partial r_{n}} \mathrm{P}(N(t)=n ; R(t) \leq r)
$$

be the density of $R(t)$ on $\{N(t)=n\}$, and on the boundary of $\Omega_{n}$ let $p(n ; r)$ be defined by continuous continuation. The support of $p(n ; r)$ is the closure $\bar{\Omega}_{n}$ of $\Omega_{n}$. Denoting by $\delta_{n}$ the set of all permutations of the set $\{1, \ldots, n\}$, from (1.3) and (1.4) for $n \in \mathbb{N}$ and $r \in \Omega_{n}$, we obtain

$$
\begin{aligned}
p(n ; r) & =\sum_{\pi \in \wp_{n}} \frac{\partial^{n}}{\partial r_{1} \cdots \partial r_{n}} \mathrm{P}\left(N(t)=n ; R_{1}^{*}(t) \leq r_{\pi(1)}, \ldots, R_{n}^{*}(t) \leq r_{\pi(n)}\right) \\
& =n ! p(n) \prod_{\ell=1}^{n} b_{R}\left(r_{\ell}\right)
\end{aligned}
$$

By continuous continuation, it follows that (2.2) also holds for $n \in \mathbb{N}$ and $r \in \bar{\Omega}_{n}$.

\subsection{Partial differential equations for LSTs of sojourn times}

Let $V_{\ell}(t), \ell=1, \ldots, N(t)$, be the sojourn time of the request with residual service time $R_{\ell}(t)$ from time $t$ to its departure (finish of service), i.e. its prospective sojourn time from time $t$ onwards. Since the $R_{\ell}(t)$ are ordered increasingly, the SDPS discipline implies that the $V_{\ell}(t)$ are also ordered increasingly, i.e. $0 \leq V_{1}(t) \leq \cdots \leq V_{N(t)}(t)$. Furthermore, $V_{1}(t)=0$ if and only if $R_{1}(t)=0$. In view of (A1) and the distributional and independence assumptions, for $0<m \leq n$ and $r \in \bar{\Omega}_{n}$, the LSTs

$$
h_{n, m}(s ; r):=\mathrm{E}\left[\mathrm{e}^{-s V_{m}(t)} \mid N(t)=n, R(t)=r\right]
$$

of $V_{m}(t)$ conditioned such that at time $t$ there are $n$ requests in the system with residual service times $R_{\ell}(t)=r_{\ell}, \ell=1, \ldots, n$, are well defined for $s \in \mathbb{R}_{+}$. Note that, for $0<m \leq n$ and $r \in \bar{\Omega}_{n}$, it holds that $h_{n, m}(0 ; r)=1$. In the following let $s \in \mathbb{R}_{+}$be fixed. As the prospective sojourn time of a request with residual service time zero is zero, from (2.3) we conclude, for $0<n$ and $r \in \bar{\Omega}_{n}$, where $r_{1}=0$, that

$$
h_{n, 1}\left(s ; 0, r_{2}, \ldots, r_{n}\right)=1
$$

Since a request with residual service time zero leaves the system immediately, for $1<m \leq n$ and $r \in \bar{\Omega}_{n}$, where $r_{1}=0$, it follows that

$$
h_{n, m}\left(s ; 0, r_{2}, \ldots, r_{n}\right)=h_{n-1, m-1}\left(s ; r_{2}, \ldots, r_{n}\right)
$$

Now let $0<m \leq n$ and $r \in \Omega_{n}$, and consider the time interval $[0, h]$. In view of $r_{1}>0$, for sufficiently small $h$, there will be no departure of any of the $n$ requests which are in the system at $t=0$. If there is a request arrival during $[0, h]$ then there may occur two cases for the sampled service time $\tau>0$, which is just its residual service time: $\tau \in\left(0, r_{m}\right)$ or $\tau \in\left[r_{m}, \infty\right)$. 
Taking into account the dynamics of the M/GI/SDPS system during $[0, h]$, we obtain

$$
\begin{gathered}
h_{n, m}(s ; r)=(1-\lambda h) \mathrm{E}\left[\mathrm{e}^{-s\left(V_{m}(h)+h\right)} \mid N(h)=n, R(h)=r-\varphi(n) h \mathbf{1}_{n}\right] \\
+\lambda h \sum_{\ell=1}^{m} \int_{r_{\ell-1}}^{r_{\ell}} b(\tau) \mathrm{E}\left[\mathrm{e}^{-s\left(V_{m+1}(h)+h\right)} \mid N(h)=n+1,\right. \\
\left.R(h)=r^{(\ell)}(r, \tau)-\varphi(n+1) h \mathbf{1}_{n+1}\right] \mathrm{d} \tau \\
+\lambda h \sum_{\ell=m+1}^{n+1} \int_{r_{\ell-1}}^{r_{\ell}} b(\tau) \mathrm{E}\left[\mathrm{e}^{-s\left(V_{m}(h)+h\right)} \mid N(h)=n+1,\right. \\
\left.R(h)=r^{(\ell)}(r, \tau)-\varphi(n+1) h \mathbf{1}_{n+1}\right] \mathrm{d} \tau+o(h),
\end{gathered}
$$

where $r_{0}:=0, r_{n+1}:=\infty, \mathbf{1}_{n}:=(1, \ldots, 1) \in \mathbb{R}^{n}$, and

$$
r^{(\ell)}(r, \tau):=\left(r_{1}, \ldots, r_{\ell-1}, \tau, r_{\ell}, \ldots, r_{n}\right), \quad \ell=1, \ldots, n+1,
$$

for $r \in \mathbb{R}^{n}$ and $\tau \in \mathbb{R}$. Subtracting $h_{n, m}\left(s ; r-\varphi(n) h \mathbf{1}_{n}\right)$ on both sides, dividing by $h$, and taking the limit $h \downarrow 0$ provides the following linear system of PDEs for $0<m \leq n$ and $r \in \Omega_{n}$ :

$$
\begin{aligned}
\left.\varphi(n) \frac{\partial}{\partial \xi} h_{n, m}\left(s ; r+\xi \mathbf{1}_{n}\right)\right|_{\xi=0} \\
=-(\lambda+s) h_{n, m}(s ; r)+\lambda \sum_{\ell=1}^{m} \int_{r_{\ell-1}}^{r_{\ell}} b(\tau) h_{n+1, m+1}\left(s ; r^{(\ell)}(r, \tau)\right) \mathrm{d} \tau \\
\quad+\lambda \sum_{\ell=m+1}^{n+1} \int_{r_{\ell-1}}^{r_{\ell}} b(\tau) h_{n+1, m}\left(s ; r^{(\ell)}(r, \tau)\right) \mathrm{d} \tau .
\end{aligned}
$$

The following observation will be crucial and considerably simplifies the analysis of the model. In view of the SDPS discipline, for $0<m \leq n$ and $r \in \bar{\Omega}_{n}$, the conditional sojourn time $V_{m}(t)$ given that $N(t)=n$ and $R(t)=r$ depends only on $r_{1}, \ldots, r_{m}$ and the total number, $n$, of requests in the system, since the requests with residual service times $r_{m+1}, \ldots, r_{n}$ have residual service times of an amount greater than or equal to $r_{m}$ and are thus in the system at least as long as the request with service time $r_{m}$. Therefore,

$$
f_{n, m}\left(s ; r_{1}, \ldots, r_{m}\right):=h_{n, m}\left(s ; r_{1}, \ldots, r_{n}\right), \quad 0<m \leq n, r \in \bar{\Omega}_{n},
$$

is well defined. From (2.4)-(2.7) we obtain the following linear system of PDEs for $0<m \leq n$ and $r \in \Omega_{m}$ :

$$
\begin{aligned}
\left.\varphi(n) \frac{\partial}{\partial \xi} f_{n, m}\left(s ; r+\xi \mathbf{1}_{m}\right)\right|_{\xi=0} \\
=-(\lambda+s) f_{n, m}(s ; r)+\lambda \sum_{\ell=1}^{m} \int_{r_{\ell-1}}^{r_{\ell}} b(\tau) f_{n+1, m+1}\left(s ; r^{(\ell)}(r, \tau)\right) \mathrm{d} \tau \\
\quad+\lambda \bar{B}\left(r_{m}\right) f_{n+1, m}(s ; r)
\end{aligned}
$$

with the initial conditions

$$
\begin{aligned}
& f_{n, 1}(s ; 0)=1, \\
& f_{n, m}\left(s ; 0, r_{2}, \ldots, r_{m}\right)=f_{n-1, m-1}\left(s ; r_{2}, \ldots, r_{m}\right), \quad 1<m \leq n,
\end{aligned}
$$


for $0 \leq r_{2} \leq \cdots \leq r_{m}$. In view of $h_{n, m}(0 ; r)=1$ for $r \in \bar{\Omega}_{n}$, from (2.7) we find that $f_{n, m}(0 ; r)=1$ for $0<m \leq n$ and $r \in \bar{\Omega}_{m}$.

It seems that, for general $\varphi(n), n \in \mathbb{N}$, there is no explicit solution to (2.8)-(2.10). However, for $\varphi_{1, k}(n)=1 /(n+k), n \in \mathbb{N}, k \in(-1, \infty)$, a solution can be given by adopting results of [13] and [22], leading to the well-known results for M/GI/1-PS systems. Note that, for $k \in \mathbb{Z}_{+}$, we have a single-server PS system with $k$ permanent requests.

Example 2.1. Let $\varphi_{1, k}(n)=1 /(n+k), n \in \mathbb{N}, k \in(-1, \infty)$, and $s \in \mathbb{R}_{+}$. We try (see [13] and [20]) the substitution

$$
f_{n, m}\left(s ; r_{1}, \ldots, r_{m}\right)=\delta\left(s, r_{m}\right)^{n+k} \prod_{i=1}^{m-1} \frac{1}{\delta\left(s, r_{m}-r_{i}\right)}
$$

for $0<m \leq n$ and $0 \leq r_{1} \leq \cdots \leq r_{m}$, where $\delta(s, \tau)$ is a continuously differentiable function in $\tau \in \mathbb{R}_{+}$with initial condition $\delta(s, 0)=1$. The substitution satisfies (2.9) and (2.10). Inserting the substitution into (2.8) and using the fact that $\varphi_{1, k}(n)=1 /(n+k), n \in \mathbb{N}$, we find after some algebra that the linear system of PDEs (2.8) is fulfilled if $\delta(s, \tau)$ satisfies the following differential equation:

$$
0=\frac{\partial \delta(s, \tau)}{\partial \tau}+\left(s+\lambda-\lambda \int_{0}^{\tau} \frac{\delta(s, \tau)}{\delta(s, \tau-y)} b(y) \mathrm{d} y-\lambda \delta(s, \tau) \bar{B}(\tau)\right) \delta(s, \tau)
$$

with initial condition $\delta(s, 0)=1$, which has a uniquely determined solution; see [20]. The product form solution for the $f_{n, m}\left(s ; r_{1}, \ldots, r_{m}\right)$, given above, has been proved for M/GI/1-PS systems for the first time by a decomposition of the sojourn time in [13] and [20], and for the single-server PS model with permanent requests in [19]. The differential equation for $\delta(s, \tau)$, given above, can be solved explicitly for particular cases. In the general case, the Laplace transform for $\mathrm{e}^{-(s+\lambda) \tau} / \delta(s, \tau)$ can be given explicitly; see [13] and [20].

For notational convenience, in the following we suppress the time parameter $t$ for steady state random variables, i.e. we use $V_{m}, N$, and $R=\left(R_{1}, \ldots, R_{N}\right)$ instead of $V_{m}(t), N(t)$, and $R(t)=\left(R_{1}(t), \ldots, R_{N(t)}(t)\right)$.

In view of (2.2) and (2.3), for $0<m \leq n$ and $r \in \Omega_{n}$, the LSTs

$$
v_{n, m}(s ; r):=\frac{\partial^{n}}{\partial r_{1} \cdots \partial r_{n}} \mathrm{E}\left[\mathrm{e}^{-s V_{m}} 1\{N=n, R \leq r\}\right]
$$

of $V_{m}$ on $\left\{N=n, R_{1} \in \mathrm{d} r_{1}, \ldots, R_{n} \in \mathrm{d} r_{n}\right\}$ are well defined for $s \in \mathbb{R}_{+}$, where $1\{\cdot\}$ denotes the indicator function, and in view of (2.2) and (2.7) are given by

$$
v_{n, m}(s ; r)=u_{n, m}\left(s ; r_{1}, \ldots, r_{m}\right) \prod_{\ell=m+1}^{n} b_{R}\left(r_{\ell}\right)
$$

where

$$
u_{n, m}\left(s ; r_{1}, \ldots, r_{m}\right):=n ! p(n)\left(\prod_{\ell=1}^{m} b_{R}\left(r_{\ell}\right)\right) f_{n, m}\left(s ; r_{1}, \ldots, r_{m}\right) .
$$

For $0<m \leq n$, let $u_{n, m}(s ; r)$ and, hence, $v_{n, m}(s ; r)$ be defined on the boundary of $\Omega_{n}$ by continuous continuation. Note that, for $0<m \leq n$ and $r \in \Omega_{n}$, from (2.11)-(2.13), it follows 
that

$$
\begin{aligned}
& u_{n, m}\left(s ; r_{1}, \ldots, r_{m}\right) \\
& \quad=\frac{\partial^{m}}{\partial r_{1} \cdots \partial r_{m}} \mathrm{E}\left[\mathrm{e}^{-s V_{m}} 1\left\{N=n, R_{1} \leq r_{1}, \ldots, R_{m} \leq r_{m}\right\} \mid R_{m+1}=r_{m+1}, \ldots, R_{n}=r_{n}\right],
\end{aligned}
$$

and, thus, the conditional LST on the right-hand side of (2.14) is independent of $r_{m+1}, \ldots, r_{n}$. From $f_{n, m}(0 ; r)=1$ and $(2.13)$, we obtain the boundary condition

$$
u_{n, m}(0 ; r)=n ! p(n) \prod_{\ell=1}^{m} b_{R}\left(r_{\ell}\right), \quad 0<m \leq n, r \in \bar{\Omega}_{m} .
$$

In the following let $s \in \mathbb{R}_{+}$be fixed. Taking the derivative of $u_{n, m}\left(s ; r+\xi \mathbf{1}_{m}\right)$ with respect to $\xi$ and taking into account (2.13), (1.2) and (1.4), we find after some algebra that (2.8) is equivalent to the following linear system of PDEs for $0<m \leq n$ and $r \in \Omega_{m}$ :

$$
\begin{aligned}
\left.\varphi(n) \frac{\partial}{\partial \xi} u_{n, m}\left(s ; r+\xi \mathbf{1}_{m}\right)\right|_{\xi=0}=- & \lambda+s+\varphi(n) \beta(r)) u_{n, m}(s ; r) \\
& +\varphi(n+1) \sum_{\ell=1}^{m} \int_{r_{\ell-1}}^{r_{\ell}} \beta(\tau) u_{n+1, m+1}\left(s ; r^{(\ell)}(r, \tau)\right) \mathrm{d} \tau \\
& +\varphi(n+1) b_{R}\left(r_{m}\right) u_{n+1, m}(s ; r)
\end{aligned}
$$

where $\beta(r):=\sum_{\ell=1}^{m} \beta\left(r_{\ell}\right)$. In view of (2.13), (1.2), and $b_{R}(0)=1 / m_{S}$, the initial conditions (2.9) and (2.10) yield the initial conditions

$$
\begin{aligned}
u_{n, 1}(s ; 0) & =n ! p(n) m_{S}^{-1}, \\
u_{n, m}\left(s ; 0, r_{2}, \ldots, r_{m}\right) & =\frac{\lambda}{\varphi(n)} u_{n-1, m-1}\left(s ; r_{2}, \ldots, r_{m}\right)
\end{aligned}
$$

for $1<m \leq n$ and $0 \leq r_{2} \leq \cdots \leq r_{m}$.

\subsection{Preliminary results}

For deriving later expressions for the LSTs of $V(n, \tau)$ and $V(\tau)$, we consider the LSTs

$$
g_{n, m}(s, x):=\frac{\partial}{\partial x} \mathrm{E}\left[\mathrm{e}^{-s V_{m}} 1\left\{N=n, R_{m} \leq x\right\}\right]
$$

for $x, s \in \mathbb{R}_{+}$and $0<m \leq n$. From (2.12) and $b_{R}(x)=\bar{B}(x) / m_{S}$, by integrating $v_{n, m}(s ; r)$ over $0 \leq r_{1} \leq \cdots \leq r_{m-1} \leq x \leq r_{m+1} \leq \cdots \leq r_{n}$ with respect to $\mathrm{d} r_{1} \cdots \mathrm{d} r_{m-1} \mathrm{~d} r_{m+1} \cdots \mathrm{d} r_{n}$ we obtain

$$
g_{n, m}(s, x)=\bar{g}_{n, m}(s, x) d_{n-m}(x),
$$

where

$$
\begin{gathered}
\bar{g}_{n, m}(s, x):=\int_{0 \leq r_{1} \leq \cdots \leq r_{m-1} \leq x} u_{n, m}\left(s ; r_{1}, \ldots, r_{m-1}, x\right) \mathrm{d} r_{1} \cdots \mathrm{d} r_{m-1}, \\
d_{\ell}(x):=\frac{1}{\ell !}\left(\int_{x}^{\infty} b_{R}(\eta) \mathrm{d} \eta\right)^{\ell}=\frac{1}{\ell !} \bar{B}_{R}(x)^{\ell} .
\end{gathered}
$$


Note that in the case in which $n=m=1$ the right-hand side of (2.19) is just $v_{1,1}(s ; x)$, and in the case in which $1=m \leq n$ the right-hand side of (2.21) is just $u_{n, 1}(s ; x)$. From (2.21), (2.15), (2.17), we find for $0<m \leq n$ that

$$
\begin{aligned}
& \bar{g}_{n, m}(0, x)=n ! p(n) b_{R}(x) \frac{1}{(m-1) !} B_{R}(x)^{m-1}, \quad x \in \mathbb{R}_{+}, \\
& \bar{g}_{n, m}(s, 0)=1\{m=1\} n ! p(n) m_{S}^{-1}, \quad s \in \mathbb{R}_{+},
\end{aligned}
$$

and, thus, from (2.20) and (2.22), we find, for $0<m \leq n$, the boundary condition

$$
g_{n, m}(s, 0)=1\{m=1\} n p(n) m_{S}^{-1}, \quad s \in \mathbb{R}_{+} .
$$

Because of (2.20), (2.22), and (2.23), we obtain, for $0<m \leq n$ and $x \in \mathbb{R}_{+}$,

$$
\begin{aligned}
g_{n, m}^{(0)}(x) & :=g_{n, m}(0, x) \\
& =n p(n) b_{R}(x)\left(\begin{array}{c}
n-1 \\
m-1
\end{array}\right) B_{R}(x)^{m-1} \bar{B}_{R}(x)^{n-m},
\end{aligned}
$$

and, thus, taking into account (1.2) and (1.4), it follows that

$$
\sum_{m=1}^{n} g_{n, m}^{(0)}(x)=n p(n) b_{R}(x)=\frac{\lambda \bar{B}(x)}{\varphi(n)} p(n-1) .
$$

For deriving later expressions and estimates for the moments of $V(n, \tau)$ and $V(\tau)$, we need some preliminary results for $g_{n, m}(s, x)$ and its derivatives with respect to $s$. For $s \in(0, \infty)$, $0<m \leq n, x \in \mathbb{R}_{+}$, and $k \in \mathbb{Z}_{+}$, let

$$
\begin{aligned}
g_{n, m}^{(k)}(s, x) & :=(-1)^{k} \frac{\partial^{k}}{\partial s^{k}} g_{n, m}(s, x) \\
& =\frac{\partial}{\partial x} \mathrm{E}\left[V_{m}^{k} \mathrm{e}^{-s V_{m}} 1\left\{N=n, R_{m} \leq x\right\}\right],
\end{aligned}
$$

where the last equality follows in view of (2.19). Note that

$$
g_{n, m}^{(k)}(s, x)=\lim _{h \downarrow 0} \mathrm{E}\left[V_{m}^{k} \mathrm{e}^{-s V_{m}} 1\left\{N=n, x<\mathbb{R}_{m} \leq x+h\right\}\right] / h \geq 0 .
$$

From (2.20) and (2.24), we find that

$$
g_{n, m}^{(k)}(s, 0)=0, \quad k \in \mathbb{N} .
$$

Taking into account $v^{k} \mathrm{e}^{-s v} \leq k ! s^{-k}$ for $v \in \mathbb{R}_{+}$, from (2.29) and (2.25), we obtain, for $s \in(0, \infty)$ and $x \in \mathbb{R}_{+}$,

$$
g_{n, m}^{(k)}(s, x) \leq k ! s^{-k} g_{n, m}^{(0)}(x), \quad 0<m \leq n, k \in \mathbb{Z}_{+} .
$$

Lemma 2.1. Let $\mathrm{E} N<\infty$. For $s \in(0, \infty)$ and $k \in \mathbb{Z}_{+}$, it holds that

$$
\sum_{0<m \leq n} \varphi(n) \frac{\partial}{\partial x} g_{n, m}^{(k)}(s, x)=-\sum_{0<m \leq n}(\varphi(n) \beta(x)+s) g_{n, m}^{(k)}(s, x)+k \sum_{0<m \leq n} g_{n, m}^{(k-1)}(s, x) .
$$


Proof. First we will prove (2.31) for $k=0$. Let $s \in[0, \infty)$ be fixed. Replacing the variables $r_{i}$ in (2.16) by $r_{i}+\eta$ for $i=1, \ldots, m$ in the arguments, integrating over $\left[-r_{1}, 0\right]$ with respect to $\eta$, and applying (2.17) and (2.18), we find that the system of PDEs (2.16) with initial conditions (2.17) and (2.18) is equivalent to the following linear system of integral equations for $0<m \leq n$ and $r \in \bar{\Omega}_{m}$ :

$$
\begin{aligned}
\varphi(n) u_{n, m}(s ; r)= & 1\{m=1\} \varphi(n) n ! p(n) m_{S}^{-1}+1\{m>1\} \lambda u_{n-1, m-1}\left(s ; r_{2}-r_{1}, \ldots, r_{m}-r_{1}\right) \\
& -\int_{-r_{1}}^{0}\left(\lambda+s+\varphi(n) \beta\left(r+\eta \mathbf{1}_{m}\right)\right) u_{n, m}\left(s ; r+\eta \mathbf{1}_{m}\right) \mathrm{d} \eta \\
+ & \varphi(n+1) \\
& \times \sum_{\ell=1}^{m} \int_{-r_{1}}^{0} \int_{r_{\ell-1}}^{r_{\ell}} \beta(\tau+\eta) u_{n+1, m+1}\left(s ; r^{(\ell)}\left(r+\eta \mathbf{1}_{m}, \tau+\eta\right)\right) \mathrm{d} \tau \mathrm{d} \eta \\
+ & \varphi(n+1) \int_{-r_{1}}^{0} b_{R}\left(r_{m}+\eta\right) u_{n+1, m}\left(s ; r+\eta \mathbf{1}_{m}\right) \mathrm{d} \eta .
\end{aligned}
$$

Integrating both sides of (2.32) over $0 \leq r_{1} \leq \cdots \leq r_{m-1} \leq x$ with respect to $\mathrm{d} r_{1} \cdots \mathrm{d} r_{m-1}$ for fixed $x=r_{m}$, using Fubini's theorem, and taking into account (2.21), we obtain after some algebra, for $0<m \leq n$ and $x \in \mathbb{R}_{+}$,

$$
\begin{aligned}
\varphi(n) \bar{g}_{n, m}(s, x)= & 1\{m=1\} \varphi(n) n ! p(n) m_{S}^{-1} \\
& +1\{m>1\} \lambda \int_{0}^{x} \bar{g}_{n-1, m-1}(s, \eta) \mathrm{d} \eta-(\lambda+s) \int_{0}^{x} \bar{g}_{n, m}(s, \eta) \mathrm{d} \eta \\
& -\varphi(n) \sum_{\ell=1}^{m-1} \int_{0}^{x} J_{n, m, \ell}(s, \eta) \mathrm{d} \eta-\varphi(n) \int_{0}^{x} \beta(\eta) \bar{g}_{n, m}(s, \eta) \mathrm{d} \eta \\
& +\varphi(n+1) \sum_{\ell=1}^{m} \int_{0}^{x} J_{n+1, m+1, \ell}(s, \eta) \mathrm{d} \eta \\
& +\varphi(n+1) \int_{0}^{x} b_{R}(\eta) \bar{g}_{n+1, m}(s, \eta) \mathrm{d} \eta
\end{aligned}
$$

where

$$
J_{n, m, \ell}(s, x):=\int_{0 \leq r_{1} \leq \cdots \leq r_{m-1} \leq x} \beta\left(r_{\ell}\right) u_{n, m}\left(s ; r_{1}, \ldots, r_{m-1}, x\right) \mathrm{d} r_{1} \cdots \mathrm{d} r_{m-1}
$$

for $0<\ell<m \leq n$ and $x \in \mathbb{R}_{+}$. In view of $\mathrm{d} d_{\ell}(x) / \mathrm{d} x=-b_{R}(x) d_{\ell-1}(x)$ for $\ell \in \mathbb{N}$ (see (2.22) and (1.4)), from (2.33) and (2.20), after some algebra, we find the following system of differential equations for the $g_{n, m}(s, x), 0<m \leq n$ and $x \in \mathbb{R}_{+}$:

$$
\begin{aligned}
\varphi(n) \frac{\partial}{\partial x} g_{n, m}(s, x) \\
=1\{m>1\} \lambda g_{n-1, m-1}(s, x)-\left(\lambda+s+\varphi(n) \beta(x)+\varphi(n)(n-m) \beta_{R}(x)\right) g_{n, m}(s, x) \\
\quad-\varphi(n) \sum_{\ell=1}^{m-1} J_{n, m, \ell}(s, x) d_{n-m}(x)+\varphi(n+1) \sum_{\ell=1}^{m} J_{n+1, m+1, \ell}(s, x) d_{n-m}(x) \\
\quad+\varphi(n+1)(n+1-m) \beta_{R}(x) g_{n+1, m}(s, x) .
\end{aligned}
$$


Now let $x^{*} \in \mathbb{R}_{+}$be arbitrary but fixed, and let

$$
\beta^{*}:=\sup \left\{\beta(x): 0 \leq x \leq x^{*}\right\},
$$

which is finite in view of (A1). Then, from (2.34) and (2.20), we find that, for $0<\ell<m \leq n$ and $0 \leq x \leq x^{*}$, it holds

$$
J_{n, m, \ell}(s, x) d_{n-m}(x) \leq \beta^{*} g_{n, m}(s, x) .
$$

Because of (2.30) for $k=0,(2.27),(2.36)$, and E $N<\infty$, summing (2.35) over $0<m \leq$ $n \leq n^{\prime}$, taking the limit $n^{\prime} \rightarrow \infty$, we find after some algebra that most of the summands cancel each other, and we obtain (2.31) for $x \in\left[0, x^{*}\right]$. Since $x^{*}$ was chosen arbitrarily, (2.31) is valid for $x \in \mathbb{R}_{+}$, completing the proof for $k=0$.

To prove (2.31) for $k \in \mathbb{N}$, we start with (2.31) for $k=0$. In view of $\left|g_{n, m}(s, x)\right| \leq g_{n, m}(0, x)$ for $\operatorname{Re}(s) \geq 0$, we may take the $k$ th derivative on both sides of (2.31) with respect to $s$ for $\operatorname{Re}(s)>0$ item by item due to Weierstrass's theorem (see [1]), which provides (2.31) for $s \in(0, \infty)$ and $k \in \mathbb{N}$.

\subsection{LSTs of sojourn times}

Consider the M/GI/SDPS system in steady state.

Theorem 2.1. For the M/GI/SDPS system, let the stability condition (1.1) and (A1) be satisfied. Then, for $s \in \mathbb{R}_{+}$and $\tau \in \mathbb{R}_{+}$, the LSTs of $V(n, \tau), n \in \mathbb{Z}_{+}$, and $V(\tau)$ are given by

$$
\begin{aligned}
\mathrm{E}\left[\mathrm{e}^{-s V(n, \tau)}\right] & =\frac{\varphi(n+1)}{\lambda(\tau) p(n)} \sum_{m=1}^{n+1} g_{n+1, m}(s, \tau), \\
\mathrm{E}\left[\mathrm{e}^{-s V(\tau)}\right] & =\frac{1}{\lambda(\tau)} \sum_{n=1}^{\infty} \varphi(n) \sum_{m=1}^{n} g_{n, m}(s, \tau),
\end{aligned}
$$

respectively, where

$$
\lambda(x):=\lambda \bar{B}(x), \quad x \in \mathbb{R}_{+},
$$

and the $g_{n, m}(s, x), 0<m \leq n, x \in \mathbb{R}_{+}$, are given by (2.20)-(2.22).

Proof. Consider a tagged arriving $\tau$-request finding $n$ requests at its arrival. From the PASTA property and conditioning with respect to the vector $R=r \in \bar{\Omega}_{n}$ of residual service times, we obtain, from (2.2), (2.3), (2.7), and (2.13),

$$
\begin{aligned}
& \mathrm{E}\left[\mathrm{e}^{-s V(n, \tau)}\right] \\
& =\frac{1}{p(n)} \sum_{m=1}^{n+1} \int_{0 \leq r_{1} \leq \cdots \leq r_{m-1} \leq \tau \leq r_{m} \leq \cdots \leq r_{n}} p(n ; r) h_{n+1, m}\left(s ; r^{(m)}(r, \tau)\right) \mathrm{d} r_{1} \cdots \mathrm{d} r_{n} \\
& =\frac{1}{(n+1) p(n+1) b_{R}(\tau)} \sum_{m=1}^{n+1} \int_{0 \leq r_{1} \leq \cdots \leq r_{m-1} \leq \tau} u_{n+1, m}\left(s ; r_{1}, \ldots, r_{m-1}, \tau\right) \mathrm{d} r_{1} \cdots \mathrm{d} r_{m-1} \\
& \quad \times \int_{\tau \leq r_{m} \leq \cdots \leq r_{n}} \prod_{\ell=m}^{n} b_{R}\left(r_{\ell}\right) \mathrm{d} r_{m} \cdots \mathrm{d} r_{n} .
\end{aligned}
$$


From (2.40), assertion (2.37) follows easily in view of $b_{R}(\tau)=\bar{B}(\tau) / m_{S}$, (1.2), (2.39), integration with respect to $\mathrm{d} r_{m} \cdots \mathrm{d} r_{n}$, and (2.20)-(2.22). Assertion (2.38) follows directly from (2.37) and

$$
\mathrm{E}\left[\mathrm{e}^{-s V(\tau)}\right]=\sum_{n=0}^{\infty} p(n) \mathrm{E}\left[\mathrm{e}^{-s V(n, \tau)}\right] .
$$

From (2.38), (2.39), $\beta(\tau)=b(\tau) / \bar{B}(\tau)$, and

$$
\mathrm{E}\left[\mathrm{e}^{-s V}\right]=\int_{\mathbb{R}_{+}} \mathrm{E}\left[\mathrm{e}^{-s V(\tau)}\right] b(\tau) \mathrm{d} \tau,
$$

we immediately obtain the following representation for the LST of $V$.

Corollary 2.1. Let the stability condition (1.1) for the M/GI/SDPS system with (A1) be satisfied. Then, for $s \in \mathbb{R}_{+}$, the LST of $V$ is given by

$$
\mathrm{E}\left[\mathrm{e}^{-s V}\right]=\frac{1}{\lambda} \sum_{n=1}^{\infty} \varphi(n) \sum_{m=1}^{n} \int_{\mathbb{R}_{+}} \beta(\tau) g_{n, m}(s, \tau) \mathrm{d} \tau .
$$

Remark 2.1. Note that Theorem 2.1 and Example 2.1 provide an approach to the LSTs of $V(n, \tau), n \in \mathbb{Z}_{+}$, and $V(\tau)$ for the M/GI/1-PS system with $k \in(-1, \infty)$ permanent requests; see [15] for an analogous approach for the M/GI/1-PS system.

\section{On the moments of $V(\tau)$}

First we prove Theorem 1.1. Then we will derive useful expressions; see Remark 3.1, below, for the moments of $V(\tau)$ and $V$, respectively.

Proof of Theorem 1.1. The lower bound in (1.8) follows directly from (1.6), (1.5), and Hölder's inequality. To prove the other assertions, we first assume in steps (i) $-(\mathrm{v})$ of the proof below that $B(x)$ satisfies (A1), i.e. $B(x)$ has a continuous density and $B(x)<1$ for $x \in \mathbb{R}_{+}$.

(i) For $s \in(0, \infty)$ and $k \in \mathbb{Z}_{+}$, from Theorem 2.1 and (2.28), we obtain

$$
\lambda(x) \mathrm{E}\left[V^{k}(x) \mathrm{e}^{-s V(x)}\right]=\sum_{0<m \leq n} \varphi(n) g_{n, m}^{(k)}(s, x), \quad x \in \mathbb{R}_{+} .
$$

In view of (2.29), for $k \in \mathbb{N}$, applying Hölder's inequality to the difference quotient

$$
\mathrm{E}\left[V_{m}^{k} \mathrm{e}^{-s V_{m}} 1\left\{N=n, x<R_{m} \leq x+h\right\}\right] / h
$$

and taking the limit $h \downarrow 0$ we find that

$$
\begin{aligned}
g_{n, m}^{(k)}(s, x) \leq & \left(\frac{\partial}{\partial x} \mathrm{E}\left[\mathrm{e}^{-s V_{m}} 1\left\{N=n, R_{m} \leq x\right\}\right]\right)^{1 /(k+1)} \\
& \times\left(\frac{\partial}{\partial x} \mathrm{E}\left[V_{m}^{k+1} \mathrm{e}^{-s V_{m}} 1\left\{N=n, R_{m} \leq x\right\}\right]\right)^{k /(k+1)} \\
\leq & \left(g_{n, m}^{(0)}(x)\right)^{1 /(k+1)}\left(g_{n, m}^{(k+1)}(s, x)\right)^{k /(k+1)},
\end{aligned}
$$


where the last inequality follows from (2.29) and (2.30) for $k=0$. Using the above inequality, applying Hölder's inequality to the series and taking into account (2.27), (3.1) provides

$$
\begin{aligned}
\frac{1}{\lambda(x)} & \sum_{0<m \leq n} g_{n, m}^{(k)}(s, x) \\
& \leq \sum_{0<m \leq n}\left(\chi(n)^{k+1} \frac{\varphi(n)}{\lambda(x)} g_{n, m}^{(0)}(x)\right)^{1 /(k+1)}\left(\frac{\varphi(n)}{\lambda(x)} g_{n, m}^{(k+1)}(s, x)\right)^{k /(k+1)} \\
& \leq\left(\sum_{0<m \leq n} \chi(n)^{k+1} \frac{\varphi(n)}{\lambda(x)} g_{n, m}^{(0)}(x)\right)^{1 /(k+1)}\left(\sum_{0<m \leq n} \frac{\varphi(n)}{\lambda(x)} g_{n, m}^{(k+1)}(s, x)\right)^{k /(k+1)} \\
& =\left(\sum_{n=0}^{\infty} \chi(n+1)^{k+1} p(n)\right)^{1 /(k+1)}\left(\mathrm{E}\left[V^{k+1}(x) \mathrm{e}^{-s V(x)}\right]\right)^{k /(k+1)} .
\end{aligned}
$$

Taking the derivative on both sides of (3.1) with respect to $x$, taking into account $\lambda(x)=\lambda \bar{B}(x)$, applying Lemma 2.1, $\beta(x)=b(x) / \bar{B}(x)$, and again (3.1), we obtain, for $s \in(0, \infty)$ and $k \in \mathbb{N}$,

$$
\lambda(x) \frac{\partial}{\partial x} \mathrm{E}\left[V^{k}(x) \mathrm{e}^{-s V(x)}\right]=\sum_{0<m \leq n}\left(k g_{n, m}^{(k-1)}(s, x)-s g_{n, m}^{(k)}(s, x)\right), \quad x \in \mathbb{R}_{+} .
$$

In view of $g_{n, m}^{(k)}(s, x) \geq 0$, in the case in which $k>1$, (3.3) and (3.2), where $k$ is replaced by $k-1$ in (3.2), imply that

$$
\frac{\partial}{\partial x} \mathrm{E}\left[V^{k}(x) \mathrm{e}^{-s V(x)}\right] \leq k\left(\sum_{n=0}^{\infty} \chi(n+1)^{k} p(n)\right)^{1 / k}\left(\mathrm{E}\left[V^{k}(x) \mathrm{e}^{-s V(x)}\right]\right)^{(k-1) / k},
$$

which is equivalent to

$$
\frac{\partial}{\partial x}\left(\mathrm{E}\left[V^{k}(x) \mathrm{e}^{-s V(x)}\right]\right)^{1 / k} \leq\left(\sum_{n=0}^{\infty} \chi(n+1)^{k} p(n)\right)^{1 / k} .
$$

Note that (3.5) is also valid for $k=1$, in view of (3.3), (2.30), (2.27), and (2.39). For $k \in \mathbb{N}$, because of $\mathrm{E}\left[V^{k}(0) \mathrm{e}^{-s V(0)}\right]=0$, integrating (3.5) over $[0, \tau]$ and taking the $k$ th power yields

$$
\mathrm{E}\left[V^{k}(\tau) \mathrm{e}^{-s V(\tau)}\right] \leq \tau^{k} \sum_{n=0}^{\infty} \chi(n+1)^{k} p(n), \quad \tau \in \mathbb{R}_{+} .
$$

The limit $s \downarrow 0$ provides the upper bound in (1.8). Thus, (1.7) implies that $\mathrm{E}\left[V^{k}(\tau)\right]$ is finite for $\tau \in \mathbb{R}_{+}$.

(ii) To prove (1.9), we derive a lower bound for $\mathrm{E}\left[V^{k}(n, \tau)\right]$. Let

$$
u_{n, m}^{(k)}(s ; r):=(-1)^{k} \frac{\partial^{k}}{\partial s^{k}} u_{n, m}(s ; r)
$$

for $s \in(0, \infty), 0<m \leq n, r \in \bar{\Omega}_{m}$, and $k \in \mathbb{Z}_{+}$. Since $0 \leq v^{k} \mathrm{e}^{-s v} \leq k ! s^{-k}$ for $v \in \mathbb{R}_{+}$, from (2.14) and (2.15), we obtain

$$
0 \leq u_{n, m}^{(k)}(s ; r) \leq k ! s^{-k} u_{n, m}(0 ; r)=k ! s^{-k} n ! p(n) \prod_{\ell=1}^{m} b_{R}\left(r_{\ell}\right) .
$$


Furthermore, from (2.17) and (2.18), we find that

$$
\begin{aligned}
u_{n, 1}^{(k)}(s ; 0) & =1\{k=0\} n ! p(n) m_{S}^{-1}, \\
u_{n, m}^{(k)}\left(s ; 0, r_{2}, \ldots, r_{m}\right) & =\frac{\lambda}{\varphi(n)} u_{n-1, m-1}^{(k)}\left(s ; r_{2}, \ldots, r_{m}\right)
\end{aligned}
$$

for $1<m \leq n$ and $0 \leq r_{2} \leq \cdots \leq r_{m}$. Taking the $k$ th derivative on both sides of (2.16) with respect to $s$, multiplying the resulting equation by $(-1)^{k}$, replacing the $r_{i}$ variables by $r_{i}+\eta, i=1, \ldots, m$, multiplying both sides by

$$
C_{n}(r ; \eta):=\exp \left(\int_{0}^{\eta} c_{n}\left(r+\xi \mathbf{1}_{m}\right) \mathrm{d} \xi\right)
$$

where

$$
c_{n}(r):=\frac{\lambda+s}{\varphi(n)}+\beta(r), \quad r \in \bar{\Omega}_{m},
$$

integrating over $\left[-r_{1}, 0\right]$ with respect to $\eta$, applying partial integration to the resulting lefthand side, and applying (3.9), we obtain the following linear system of integral equations for $0<m \leq n, r \in \bar{\Omega}_{m}, s \in(0, \infty)$, and $k \in \mathbb{N}$ :

$$
\begin{aligned}
& \varphi(n) u_{n, m}^{(k)}(s ; r) \\
& =1\{m>1\} \lambda u_{n-1, m-1}^{(k)}\left(s ; r_{2}-r_{1}, \ldots, r_{m}-r_{1}\right) C_{n}\left(r ;-r_{1}\right) \\
& \quad+\int_{-r_{1}}^{0}\left(\varphi ( n + 1 ) \left(\sum_{\ell=1}^{m} \int_{r_{\ell-1}}^{r_{\ell}} \beta(\tau+\eta) u_{n+1, m+1}^{(k)}\left(s ; r^{(\ell)}\left(r+\eta \mathbf{1}_{m}, \tau+\eta\right)\right) \mathrm{d} \tau\right.\right. \\
& \left.\left.\quad+b_{R}\left(r_{m}+\eta\right) u_{n+1, m}^{(k)}\left(s ; r+\eta \mathbf{1}_{m}\right)\right)+k u_{n, m}^{(k-1)}\left(s ; r+\eta \mathbf{1}_{m}\right)\right) \\
& \quad \times C_{n}(r ; \eta) \mathrm{d} \eta .
\end{aligned}
$$

For $s \downarrow 0$, from (3.10) and (3.8), we find that, for

$$
u_{n, m}^{(k)}(r):=\lim _{s \downarrow 0} u_{n, m}^{(k)}(s ; r), \quad 0<m \leq n, r \in \bar{\Omega}_{m}, k \in \mathbb{N},
$$

it holds that

$$
\varphi(n) u_{n, m}^{(k)}(r) \geq k \int_{-r_{1}}^{0} u_{n, m}^{(k-1)}\left(r+\eta \mathbf{1}_{m}\right) C_{n}(r ; \eta) \mathrm{d} \eta .
$$

For $0<m \leq n$ and $k \in \mathbb{N}$, let

$$
\begin{aligned}
\bar{g}_{n, m}^{(k)}(x): & =\int_{0 \leq r_{1} \leq \cdots \leq r_{m-1} \leq x} u_{n, m}^{(k)}\left(r_{1}, \ldots, r_{m-1}, x\right) \mathrm{d} r_{1} \cdots \mathrm{d} r_{m-1} \\
& =(-1)^{k} \lim _{s \downarrow 0} \frac{\partial^{k}}{\partial s^{k}} \bar{g}_{n, m}(s, x) ;
\end{aligned}
$$

cf. (2.21), (3.7), and (3.11). Now let $x^{*} \in \mathbb{R}_{+}$be arbitrary but fixed, let

$$
\beta^{*}:=\sup \left\{\beta(x): 0 \leq x \leq x^{*}\right\},
$$


and let

$$
a_{n}(x):=\exp \left(-\left(\frac{\lambda}{\varphi(n)}+n \beta^{*}\right) x\right), \quad x \in \mathbb{R}_{+} .
$$

Integrating (3.12) over $0 \leq r_{1} \leq \cdots \leq r_{m-1} \leq x$ with respect to $\mathrm{d} r_{1} \cdots \mathrm{d} r_{m-1}$, taking into account

$$
C_{n}(r ;-\eta) \geq a_{n}(x), \quad 0 \leq \eta \leq r_{1} \leq \cdots \leq r_{m-1} \leq r_{m}=x \leq x^{*},
$$

and using Fubini's theorem, for $0<m \leq n$ and $k \in \mathbb{N}$, we obtain

$$
\varphi(n) \bar{g}_{n, m}^{(k)}(x) \geq k a_{n}(x) \int_{0}^{x} \bar{g}_{n, m}^{(k-1)}(\eta) \mathrm{d} \eta, \quad 0 \leq x \leq x^{*} .
$$

(iii) For $0<m \leq n, k \in \mathbb{Z}_{+}$, and $x \in \mathbb{R}_{+}$, let

$$
g_{n, m}^{(k)}(x):=\lim _{s \downarrow 0} g_{n, m}^{(k)}(s, x)=\frac{\partial}{\partial x} \mathrm{E}\left[V_{m}^{k} 1\left\{N=n, R_{m} \leq x\right\}\right] ;
$$

cf. (2.25), (2.28), and (2.29). Note that, from (2.20), (2.28), (3.13), and (3.16), it follows that

$$
g_{n, m}^{(k)}(x)=\bar{g}_{n, m}^{(k)}(x) d_{n-m}(x) .
$$

(iv) Now we will show by induction that, for $k \in \mathbb{Z}_{+}, n \in \mathbb{N}$, and $x \in\left[0, x^{*}\right]$, it holds that

$$
\varphi(n) \sum_{m=1}^{n} g_{n, m}^{(k)}(x) \geq \lambda(x) x^{k} p(n-1)\left(\frac{a_{n}(x) \bar{B}_{R}(x)^{n-1}}{\varphi(n)}\right)^{k} .
$$

For $k=0$, assertion (3.18) follows directly from (2.27) and (2.39). Assume that (3.18) is true for $k \in \mathbb{Z}_{+}$. For $k+1$, by using (3.17), (3.15), and (2.22), the induction assumption for $k$, and by taking into account the facts that $\bar{B}_{R}(\eta), a_{n}(\eta)$, and $\lambda(\eta)$ are decreasing in $\eta, \bar{B}_{R}(\eta) \leq 1$, and $\bar{B}_{R}(0)=1$, we find that

$$
\begin{aligned}
\varphi(n) & \sum_{m=1}^{n} g_{n, m}^{(k+1)}(x) \\
& \geq(k+1) a_{n}(x) \sum_{m=1}^{n} \int_{0}^{x} \bar{g}_{n, m}^{(k)}(\eta) d_{n-m}(x) \mathrm{d} \eta \\
& \geq(k+1) a_{n}(x) \int_{0}^{x} \sum_{m=1}^{n} \bar{g}_{n, m}^{(k)}(\eta) d_{n-m}(\eta) \bar{B}_{R}(x)^{n-m} \mathrm{~d} \eta \\
& \geq(k+1) a_{n}(x) \bar{B}_{R}(x)^{n-1} \int_{0}^{x} \frac{1}{\varphi(n)} \lambda(\eta) \eta^{k} p(n-1)\left(\frac{a_{n}(\eta) \bar{B}_{R}(\eta)^{n-1}}{\varphi(n)}\right)^{k} \mathrm{~d} \eta \\
& \geq \lambda(x) x^{k+1} p(n-1)\left(\frac{a_{n}(x) \bar{B}_{R}(x)^{n-1}}{\varphi(n)}\right)^{k+1},
\end{aligned}
$$

completing the induction step, i.e. (3.18) is proved.

(v) From Theorem 2.1, (2.28), (3.16), and (3.18), we obtain, for $k \in \mathbb{N}$,

$$
\mathrm{E}\left[V^{k}(n, \tau)\right] \geq \tau^{k}\left(\frac{a_{n+1}(\tau) \bar{B}_{R}(\tau)^{n}}{\varphi(n+1)}\right)^{k}, \quad \tau \in \mathbb{R}_{+}, n \in \mathbb{Z}_{+} .
$$


In view of $\lim _{\tau \downarrow 0} a_{n+1}(\tau)=\lim _{\tau \downarrow 0} \bar{B}_{R}(\tau)=1$, cf. (3.14), we find that

$$
\liminf _{\tau \downarrow 0} \frac{\mathrm{E}\left[V^{k}(n, \tau)\right]}{\tau^{k}} \geq \chi(n+1)^{k}, \quad n \in \mathbb{Z}_{+} .
$$

Multiplying both sides of (3.19) by $p(n)$, summing up over $n \in \mathbb{Z}_{+}$, and taking into account (1.8), we obtain (1.9). For fixed $n \in \mathbb{Z}_{+}$, from (3.20) and (1.9), it follows that

$$
\begin{aligned}
\underset{\tau \downarrow 0}{\limsup } & \frac{\mathrm{E}\left[V^{k}(n, \tau)\right]}{\tau^{k}} p(n)+\sum_{j \in \mathbb{Z}_{+} \backslash\{n\}} \chi(j+1)^{k} p(j) \\
& \leq \limsup _{\tau \downarrow 0} \frac{\mathrm{E}\left[V^{k}(n, \tau)\right]}{\tau^{k}} p(n)+\sum_{j \in \mathbb{Z}_{+} \backslash\{n\}} \liminf _{\tau \downarrow 0} \frac{\mathrm{E}\left[V^{k}(j, \tau)\right]}{\tau^{k}} p(j) \\
& \leq \limsup _{\tau \downarrow 0} \sum_{j \in \mathbb{Z}_{+}} \frac{\mathrm{E}\left[V^{k}(j, \tau)\right]}{\tau^{k}} p(j) \\
& =\limsup _{\tau \downarrow 0} \frac{\mathrm{E}\left[V^{k}(\tau)\right]}{\tau^{k}} \\
& =\sum_{j \in \mathbb{Z}_{+}} \chi(j+1)^{k} p(j),
\end{aligned}
$$

which provides (1.11) in view of (3.20) if (1.7) is fulfilled.

(vi) The case of a general DF $B(x)$ with finite mean $m_{S}$ is obtained by taking the limit in distribution of a sequence of service time distributions $B_{v}(x), v=1,2, \ldots$, where the $B_{v}(x)$ have the given mean $m_{S}$, fulfill (A1), and converge weakly to $B(x)$. Since the assertions hold for the $B_{v}(x)$, by arguments of continuity we obtain the assertions for $B(x)$ in view of (1.1) and (1.2).

(vii) For fixed $q \in(0,1]$, let the service time DF be given by

$$
B_{q}(x):=(1-q) 1\{x \geq 0\}+q 1\left\{x \geq \frac{m_{S}}{q}\right\}, \quad x \in \mathbb{R}_{+} .
$$

Obviously, the mean service time is $m_{S}$. Note that, under the SDPS discipline, the sojourn times of the zero service time requests are zero and they do not have any impact on the system dynamics. Thus, the dynamics of this M/GI/SDPS system correspond to those of an M/D/SDPS system with arrival rate $\lambda_{q}:=q \lambda$ and deterministic service times $m_{S} / q$. Therefore, the sojourn time $V_{q}(\tau)$ of an arriving tagged $\tau$-request in this M/GI/SDPS system equals the sojourn time of an arriving tagged $\tau$-request in the M/D/SDPS system with arrival rate $\lambda_{q}$ and deterministic service times $m_{S} / q$ in distribution. Time scaling shows that $V_{q}(\tau)$ equals $V(q \tau) / q$ in distribution, where $V(\tau)$ denotes the sojourn time of an arriving tagged $\tau$-request in the M/D/SDPS system with given arrival rate $\lambda$ and deterministic service times $m_{S}$. Thus, from (1.9), for any $k \in \mathbb{Z}_{+}$and any $\tau \in(0, \infty)$, it follows that

$$
\lim _{q \downarrow 0} \frac{\mathrm{E}\left[V_{q}^{k}(\tau)\right]}{\tau^{k}}=\lim _{q \tau \downarrow 0} \frac{\mathrm{E}\left[V^{k}(q \tau)\right]}{(q \tau)^{k}}=\sum_{n=0}^{\infty} \chi(n+1)^{k} p(n),
$$

which provides (1.10). 
Next we will derive expressions for the moments of $V(\tau)$ and $V$, respectively, which are useful in some applications; see Remark 3.1, below.

Theorem 3.1. For the M/GI/SDPS system, let the stability condition (1.1) and (A1) be satisfied, and let $k \in \mathbb{N}$ such that (1.7) is fulfilled. Then the kth moment of $V(\tau)$ is finite, and it holds that

$$
\mathrm{E}\left[V^{k}(\tau)\right]=k \sum_{n=1}^{\infty} \sum_{m=1}^{n} \int_{0}^{\tau} \frac{g_{n, m}^{(k-1)}(x)}{\lambda(x)} \mathrm{d} x, \quad \tau \in \mathbb{R}_{+},
$$

where the $g_{n, m}^{(k-1)}(x)$ are given by (2.26), (2.28), and (3.16).

Proof. In the case in which $k>1,(3.2)$, with $k$ replaced by $k-1,(3.6)$, and taking the limit $s \downarrow 0$ yield

$$
\frac{1}{\lambda(x)} \sum_{0<m \leq n} g_{n, m}^{(k-1)}(x) \leq x^{k-1} \sum_{n=0}^{\infty} \chi(n+1)^{k} p(n), \quad x>0 .
$$

Note that (3.23) also holds for $k=1$, in view of (2.27). Taking into account $x \mathrm{e}^{-x} \leq 1$ for $x \in \mathbb{R}_{+}$, from (2.29) we find that

$$
\begin{aligned}
s g_{n, m}^{(k)}(s, x) & =\lim _{h \downarrow 0} \mathrm{E}\left[V_{m}^{k-1}\left(s V_{m} \mathrm{e}^{-s V_{m}}\right) 1\left\{N=n, x<R_{m} \leq x+h\right\}\right] / h \\
& \leq g_{n, m}^{(k-1)}(x) .
\end{aligned}
$$

Moreover, from (2.38) and (1.8) we obtain

$$
\varphi(n) g_{n, m}^{(k)}(s, x) \leq \lambda(x) \mathrm{E}\left[V^{k}(x)\right] \leq \lambda(x) x^{k} \sum_{n=0}^{\infty} \chi(n+1)^{k} p(n),
$$

which implies that $\lim _{s \downarrow 0} s g_{n, m}^{(k)}(s, x)=0$. Due to (3.23) and Lebesgue's theorem, it follows that

$$
\lim _{s \downarrow 0} \frac{1}{\lambda(x)} \sum_{0<m \leq n} s g_{n, m}^{(k)}(s, x)=0, \quad x>0 .
$$

Because of (3.23) and (3.24), taking the limit $s \downarrow 0$ in (3.3) provides

$$
\frac{\mathrm{d}}{\mathrm{d} x} \mathrm{E}\left[V^{k}(x)\right]=\frac{k}{\lambda(x)} \sum_{n=1}^{\infty} \sum_{m=1}^{n} g_{n, m}^{(k-1)}(x), \quad x>0 .
$$

In view of $\mathrm{E}\left[V^{k}(0)\right]=0$, integrating over $[0, \tau]$ yields $(3.22)$.

Note that, because of (2.27), for $k=1$, from (3.22), it follows that

$$
\mathrm{E}[V(\tau)]=\tau \sum_{n=0}^{\infty} \chi(n+1) p(n), \quad \tau \in \mathbb{R}_{+},
$$

which corresponds to Cohen's general result (1.6); cf. (1.5). From (3.22) and

$$
\mathrm{E}\left[V^{k}\right]=\int_{0}^{\infty} \mathrm{E}\left[V^{k}(\tau)\right] b(\tau) \mathrm{d} \tau,
$$

via Fubini's theorem and Theorem 1.1 we obtain the following results for the unconditional sojourn time $V$. 
Theorem 3.2. For the M/GI/SDPS system, let the stability condition (1.1) and (A1) be satisfied, and let $k \in \mathbb{N}$ such that (1.7) is fulfilled and $\mathrm{E}\left[S^{k}\right]<\infty$. Then the kth moment of $V$ is finite, and it holds that

$$
\mathrm{E}\left[V^{k}\right]=\frac{k}{\lambda} \sum_{n=1}^{\infty} \sum_{m=1}^{n} \int_{0}^{\infty} g_{n, m}^{(k-1)}(x) \mathrm{d} x
$$

where the $g_{n, m}^{(k-1)}(x)$ are given by (2.26), (2.28), and (3.16).

Remark 3.1. Equations (3.22) and (3.25) are useful for a numerical computation of sojourn time characteristics for particular M/GI/SDPS systems, because they allow for a reduction in the numerical complexity (from $k$ to $k-1$ ); see [6] for the M/M/SDPS system and [8] for the M/D/SDPS system.

\section{Waiting times}

The waiting time in an M/GI/SDPS system is defined as the difference between the sojourn and required service times of a request; see [22, p. 107]. Let

$$
W:=V-S \text { and } W(\tau):=V(\tau)-\tau
$$

be the waiting time of an arbitrary arriving request and an arriving $\tau$-request, respectively. In order to ensure that the waiting times are nonnegative, we assume in the following that $0<\varphi(n) \leq 1, n \in \mathbb{N}$, and that there exists an $n \in \mathbb{N}$ such that $\varphi(n)<1$. (In the case in which $\varphi(n)=1, n \in \mathbb{N}$, the system corresponds to an $\mathrm{M} / \mathrm{GI} / \infty$ system, where no waiting occurs.) We immediately have $\mathrm{E} W=\mathrm{E} V-\mathrm{E} S$, $\operatorname{var}(W(\tau))=\operatorname{var}(V(\tau))$, and, from (1.6), $\mathrm{E} W(\tau)=(\tau / \mathrm{E} S) \mathrm{E} W$. For the variances $\operatorname{var}(V)$ and $\operatorname{var}(W)$ or, equivalently, for the squared coefficients of variation

$$
c_{V}^{2}:=\frac{\operatorname{var}(V)}{(\mathrm{E} V)^{2}} \quad \text { and } \quad c_{W}^{2}:=\frac{\operatorname{var}(W)}{(\mathrm{E} W)^{2}},
$$

it holds that

$$
(\mathrm{E} V)^{2}\left(c_{V}^{2}-c_{S}^{2}\right)=(\mathrm{E} W)^{2}\left(c_{W}^{2}-c_{S}^{2}\right)=\int_{\mathbb{R}_{+}} \operatorname{var}(W(\tau)) \mathrm{d} B(\tau) \geq 0
$$

(cf. [6, Equation (4.4)]), which implies that

$$
\operatorname{var}(V)=\operatorname{var}(W)+\operatorname{var}(S)+2 c_{S}^{2} \mathrm{E} W \mathrm{E} S .
$$

(For a random variable $X, c_{X}^{2}:=\operatorname{var}(X) /(\mathrm{E} X)^{2}$ denotes the squared coefficient of variation.) Note that, owing to the SDPS discipline, waiting and service times are not independent, in contrast to the FCFS discipline; see (4.2). Moreover, from (4.1), it follows that $c_{S}^{2} \leq c_{V}^{2} \leq c_{W}^{2}$. Note that in (4.1) the sojourn and waiting times occur symmetrically, reflecting the fact that results for sojourn times have a correspondence to results for waiting times, and vice versa. Below we shortly summarize some corresponding results for waiting times in an M/GI/SDPS system. The proofs are analogous to the arguments given in Sections 2 and 3, where we only have to take into account the fact that the waiting time increases by $(1-\varphi(n)) h$ during an interval $[0, h]$ if $n$ requests are in the system and if there is no arrival and no departure. Thus, in the corresponding equation leading to (2.6), the term $\mathrm{e}^{-s\left(V_{m}(h)+h\right)}$ has to be replaced by $\mathrm{e}^{-s\left(W_{m}(h)+(1-\varphi(n)) h\right)}$, and in (2.6) the term $\lambda+s$ has to be replaced by $\lambda+s(1-\varphi(n))$.

By paralleling the proof of Theorem 1.1, we obtain the following theorem. 
Theorem 4.1. Let the stability condition (1.1) for the M/GI/SDPS system be satisfied. Then, for $k \in \mathbb{Z}_{+}$, the kth moment of $W(\tau), \tau \in \mathbb{R}_{+}$, is finite if

$$
\sum_{n=0}^{\infty}(\chi(n+1)-1)^{k} p(n)<\infty
$$

For $k \in \mathbb{Z}_{+}$, it holds that

$$
\begin{gathered}
\tau^{k}\left(\sum_{n=0}^{\infty}(\chi(n+1)-1) p(n)\right)^{k} \leq \mathrm{E}\left[W^{k}(\tau)\right] \leq \tau^{k} \sum_{n=0}^{\infty}(\chi(n+1)-1)^{k} p(n), \quad \tau \in \mathbb{R}_{+}, \\
\lim _{\tau \downarrow 0} \frac{\mathrm{E}\left[W^{k}(\tau)\right]}{\tau^{k}}=\sum_{n=0}^{\infty}(\chi(n+1)-1)^{k} p(n) .
\end{gathered}
$$

There exists a family of service time distributions $B_{q}(x), q \in(0,1]$, with given mean $m_{S}$ such that, for the waiting times $W_{q}(\tau)$ of a tagged arriving $\tau$-request in the M/GI/SDPS system with service time distribution $B_{q}(x)$, it holds that

$$
\lim _{q \downarrow 0} \mathrm{E}\left[W_{q}^{k}(\tau)\right]=\tau^{k} \sum_{n=0}^{\infty}(\chi(n+1)-1)^{k} p(n), \quad \tau \in \mathbb{R}_{+}, k \in \mathbb{Z}_{+} .
$$

If, in addition, (4.3) is satisfied then it holds that

$$
\lim _{\tau \downarrow 0} \frac{\mathrm{E}\left[W^{k}(n, \tau)\right]}{\tau^{k}}=(\chi(n+1)-1)^{k}, \quad n \in \mathbb{Z}_{+} .
$$

\section{Numerical examples}

In terms of the squared coefficient of variation, the lower and upper bounds for $\mathrm{E}\left[V^{2}(\tau)\right]$ given in (1.8) are equivalent to

$$
0 \leq c_{V(\tau)}^{2} \leq c_{\chi(N+1)}^{2}, \quad \tau \in(0, \infty)
$$

as we have equality in (1.8) for $k=1$. Furthermore, for $k=2,(1.9)$ is equivalent to

$$
\lim _{\tau \downarrow 0} c_{V(\tau)}^{2}=c_{\chi(N+1)}^{2} .
$$

In Tables 1 and 2 an $m$-server system under processor sharing is considered, i.e. $\varphi(n)=$ $\min (m / n, 1), n \in \mathbb{N}$, where the service times are exponential in Table 1 and deterministic in Table 2. In Table $1, c_{V(\tau)}^{2}$ and the upper bound $c_{\chi(N+1)}^{2}$ in the $\mathrm{M} / \mathrm{M} / \mathrm{m}-\mathrm{PS}$ system for $m=1,2,4,8$ are given. Note that $\mathrm{E}\left[V^{2}(\tau)\right]$ has been computed for $m \neq 1$ by means of the algorithm given in [6, Equations (2.24), (2.26)-(2.28)] for M/M/SDPS systems, based on numerically solving an infinite linear system of ordinary differential equations with constant coefficients. In Table 2, $c_{V(\tau)}^{2}$ and the insensitive upper bound $c_{\chi(N+1)}^{2}$ in the M/D/m-PS system for $m=1,2$ are given, where $c_{V(\tau)}^{2}$ has been computed for the M/D/1-PS system via

$$
c_{V(\tau)}^{2}=1-\left.2(1-\varrho) \frac{\mathrm{e}^{\xi}-1-\xi}{\xi^{2}}\right|_{\xi=\lambda \tau}, \quad \tau \in\left(0, m_{S}\right],
$$


TABLE 1: The squared coefficient of variation of $V(\tau)$ in the M/M/m-PS system and its insensitive upper bound for $m=1,2,4,8$.

\begin{tabular}{|c|c|c|c|c|c|c|}
\hline \multirow{2}{*}{$\varrho / m$} & \multirow{2}{*}{$m$} & \multicolumn{4}{|c|}{$c_{V(\tau)}^{2}$} & \multirow{2}{*}{$c_{\chi(N+1)}^{2}$} \\
\hline & & $\tau / m_{S}=0.01$ & $\tau / m_{S}=0.1$ & $\tau / m_{S}=1$ & $\tau / m_{S}=10$ & \\
\hline 0.20 & 1 & 0.199468 & 0.194772 & 0.155831 & 0.043752 & 0.200000 \\
\hline 0.50 & 1 & 0.499168 & 0.491770 & 0.426123 & 0.160270 & 0.500000 \\
\hline 0.80 & 1 & 0.799467 & 0.794693 & 0.749230 & 0.454134 & 0.800000 \\
\hline 0.95 & 1 & 0.949842 & 0.948419 & 0.934363 & 0.809633 & 0.950000 \\
\hline 0.20 & 2 & 0.027085 & 0.026090 & 0.018864 & 0.004401 & 0.027200 \\
\hline 0.50 & 2 & 0.218282 & 0.214157 & 0.179580 & 0.060706 & 0.218750 \\
\hline 0.80 & 2 & 0.626740 & 0.622622 & 0.583827 & 0.341777 & 0.627200 \\
\hline 0.95 & 2 & 0.901218 & 0.899834 & 0.886176 & 0.765378 & 0.901372 \\
\hline 0.20 & 4 & 0.001099 & 0.001029 & 0.000621 & 0.000113 & 0.001107 \\
\hline 0.50 & 4 & 0.048647 & 0.047327 & 0.037349 & 0.010697 & 0.048800 \\
\hline 0.80 & 4 & 0.367796 & 0.364898 & 0.338256 & 0.184834 & 0.368122 \\
\hline 0.95 & 4 & 0.792035 & 0.790747 & 0.778059 & 0.666827 & 0.792178 \\
\hline 0.20 & 8 & 0.000008 & 0.000007 & 0.000003 & 0.000000 & 0.000008 \\
\hline 0.50 & 8 & 0.005137 & 0.004915 & 0.003479 & 0.000795 & 0.005164 \\
\hline 0.80 & 8 & 0.144923 & 0.143407 & 0.130066 & 0.063173 & 0.145096 \\
\hline 0.95 & 8 & 0.602936 & 0.601836 & 0.591056 & 0.498428 & 0.603059 \\
\hline
\end{tabular}

TABLE 2: The squared coefficient of variation of $V(\tau)$ in the M/D/m-PS system and its insensitive upper bound for $m=1,2$.

\begin{tabular}{cccccc}
\hline & & \multicolumn{5}{c}{$c_{V(\tau)}^{2}$} & \multirow{2}{*}{$c_{\chi(N+1)}^{2}$} \\
\cline { 3 - 5 }$\varrho / m$ & $m$ & & & \\
& & $\tau / m_{S}=0.01$ & $\tau / m_{S}=0.1$ & $\tau / m_{S}=1$ & \\
\hline 0.20 & 1 & 0.199466 & 0.194640 & 0.143890 & 0.200000 \\
0.50 & 1 & 0.499166 & 0.491561 & 0.405115 & 0.500000 \\
0.80 & 1 & 0.799466 & 0.794558 & 0.734037 & 0.800000 \\
0.95 & 1 & 0.949841 & 0.948378 & 0.929561 & 0.950000 \\
0.20 & 2 & 0.027085 & 0.026063 & 0.017136 & 0.027200 \\
0.50 & 2 & 0.218281 & 0.214042 & 0.169378 & 0.218750 \\
0.80 & 2 & 0.626738 & 0.622506 & 0.571023 & 0.627200 \\
0.95 & 2 & 0.901218 & 0.899795 & 0.881514 & 0.901372 \\
\hline
\end{tabular}

which can be obtained from [15, Section 5], and, for the M/D/2-PS system via

$$
\begin{aligned}
c_{V(\tau)}^{2}= & \frac{\varrho}{2}-\left(1+\frac{\varrho}{2}\right)\left(1-\frac{\varrho}{2}\right) \frac{\mathrm{e}^{\xi}-1-\xi}{\xi^{2}} \\
& +\left.\frac{1}{18}\left(1+\frac{\varrho}{2}\right)\left(1-\frac{\varrho}{2}\right)^{3} \frac{(12 \xi-10) \mathrm{e}^{\xi}+9+\mathrm{e}^{-2 \xi}}{\xi^{2}}\right|_{\xi=\lambda \tau / 2}, \quad \tau \in\left(0, m_{S}\right]
\end{aligned}
$$

cf. [8, Corollary 4.1]. 
Table 1 and Table 2 illustrate (5.1). The right-hand side of (5.1) is a good approximation for $c_{V(\tau)}^{2}$ if $\tau / m_{S}$ is small, corresponding to (5.2), or if $\varrho / m$ is close to the stability bound 1 ; cf. (1.1). The lower bound 0 for $c_{V(\tau)}^{2}$, cf. (5.1), seems to be the limit of $c_{V(\tau)}^{2}$ for $\tau \rightarrow \infty$. Note that the upper bound $c_{\chi(N+1)}^{2}$ is close to the lower bound 0 for small $\varrho$ due to continuity and since $N=0$ almost surely in the limit $\varrho \downarrow 0$. In particular, for the single-server system, i.e. for $m=1$, it holds that $c_{\chi(N+1)}^{2}=\varrho$, and, for the two-server system, i.e. for $m=2$, it holds that

$$
c_{\chi(N+1)}^{2}=\frac{1}{2}\left(\frac{\varrho}{2}\right)^{2}+\left(\frac{\varrho}{2}\right)^{3}-\frac{1}{2}\left(\frac{\varrho}{2}\right)^{4} .
$$

Note that these expressions for $c_{\chi(N+1)}^{2}$ can easily be derived by taking the limit in (5.3) and (5.4) for $\tau \downarrow 0$, respectively, due to (5.2).

Better but more complex approximations for $c_{V(\tau)}^{2}$ in M/GI/SDPS systems are given in [7]. The tables also illustrate the fact that $c_{V(\tau)}^{2}$ is sensitive with respect to the service time DF; see also [18]. Note that in case of the DF in (3.21) used for proving (1.10), the squared coefficient of variation of the service time equals $(1-q) / q$, which tends to $\infty$ for $q \downarrow 0$.

\section{References}

[1] Ahlfors, L. V. (1979). Complex Analysis. McGraw-Hill, New York.

[2] Bonald, T. ANd Proutière, A. (2002). Insensitivity in processor-sharing networks. Performance Evaluation 49, 193-209.

[3] Borst, S., Boxma, O. And Jelenković, P. (2003). Reduced-load equivalence and induced burstiness in GPS queues with long-tailed traffic flows. Queueing Systems 43, 273-306.

[4] Borst, S., Mandjes, M. And van Uitert, M. (2003). Generalized processor sharing queues with heterogeneous traffic classes. Adv. Appl. Prob. 35, 806-845.

[5] Braband, J. (1994). Waiting time distributions for $M / M / N$ processor sharing queues. Commun. Statist. Stoch. Models 10, 533-548.

[6] Brandt, A. And Brandt, M. (2008). Waiting times for $M / M$ systems under state-dependent processor sharing. Queueing Systems 59, 297-319.

[7] Brandt, A. And Brandt, M. (2009). Approximations for the second moments of sojourn times in $M / G I$ systems under state-dependent processor sharing. ZIB Rep. 09-25, Konrad-Zuse-Zentrum für Informationstechnik Berlin. Available at http://opus.kobv.de/zib/volltexte/2009/1190/.

[8] Brandt, M. and Brandt, A. (2010). On sojourn times in $M / G I$ systems under state-dependent processor sharing. Queueing Systems 64, 167-201.

[9] Cheung, S.-K., van den Berg, H. and Boucherie, R. (2006). Insensitive bounds for the moments of the sojourn time distribution in the $M / G / 1$ processor-sharing queue. Queueing Systems 53, 7-18.

[10] Coffman, E. G., JR. Muntz, R. R. and Trotter, H. (1970). Waiting time distributions for processor-sharing systems. J. Assoc. Comput. Mach. 17, 123-130.

[11] CoHen, J. W. (1979). The multiple phase service network with generalized processor sharing. Acta Informatica 12, 245-284.

[12] Guillemin, F., Robert, P. And Zwart, B. (2004). Tail asymptotics for processor-sharing queues. Adv. Appl. Prob. 36, 525-543.

[13] Kitaev, M. Y. and Yashrov, S. F. (1978). Distribution of the conditional sojourn time of requests in sharedprocessor systems. Izv. Akad. Nauk SSSR Tekh. Kibernet. 4, 211-215.

[14] Kleinrock, L. (1967). Time-shared systems: a theoretical treatment. J. Assoc. Comput. Mach. 14, $242-261$.

[15] Отт, T. J. (1984). The sojourn-time distribution in the $M / G / 1$ queue with processor sharing. J. Appl. Prob. 21, 360-378.

[16] Parekh, A. K. and Gallager, R. G. (1993). A generalized processor sharing approach to flow control in integrated services networks: the single-node case. IEEE/ACM Trans. Networking 1, 344-357.

[17] Tolmachev, A. L. (1987). Insensitivity in queueing networks with generalized processor-sharing discipline. In Proc. 1st World Congr. Bernoulli Soc. (Tashkent, 1986), Vol. 1, VNU Science Press, Utrecht, pp. 283-286.

[18] Van den Berg, J. L. And Boxma, O. J. (1991). The $M / G / 1$ queue with processor sharing and its relation to a feedback queue. Queueing Systems 9, 365-401.

[19] Yashkova, A. S. AND YashKov, S. F. (2003). Distribution of the virtual sojourn time in the $M / G / 1$ processor sharing queue. Inf. Process. 3, 128-137. 
[20] Yashkov, S. F. (1983). A derivation of response time distribution for an $M / G / 1$ processor-sharing queue. Prob. Control Inf. Theory 12, 133-148.

[21] YASHKov, S. F. (1987). Processor-sharing queues: some progress in analysis. Queueing Systems 2, 1-17.

[22] Yashkov, S. F. (1992). Mathematical problems in the theory of processor-sharing systems. J. Soviet Math. 58, $101-147$.

[23] Yashkov, S. F. And Yashkova, A. S. (2007). Processor sharing: a survey of the mathematical theory. Automation Remote Control 68, 1662-1731.

[24] Zachary, S. (2007). A note on insensitivity in stochastic networks. J. Appl. Prob. 44, 238-248.

[25] Zhang, Z. L., Towsley, D. And Kurose, J. (1994). Statistical analysis of generalized processor sharing scheduling discipline. ACM SIGCOMM Comput. Commun. Rev. 24, 68-77. 